\title{
Reply to "Indications and timing for tracheostomy in patients with SARS CoV2-related" by Ferri et al.
}

\author{
Francesco Mattioli ${ }^{1} \cdot$ Andrea Marudi $^{2} \cdot$ Michael Ghirelli $^{1} \cdot$ Gabriele Molteni $^{3} \cdot$ Nicola Sgarbi $^{2} \cdot$ Sara Valerini $^{1}$. \\ Massimo Girardis ${ }^{2} \cdot$ Livio Presutti $^{1} \cdot$ Matteo Fermi $^{1}$ (i)
}

Received: 4 June 2020 / Accepted: 12 June 2020 / Published online: 17 June 2020

(c) Springer-Verlag GmbH Germany, part of Springer Nature 2020

\section{Dear Editor,}

We sincerely appreciate the interest of Ferri et al. in our study and we are grateful for the valuable comments and feedback which have allowed us to respond with some clarifications [1]. The Letter to the Editor by Ferri et al. is merely focused on the timing of the tracheostomy, which actually is not the main aspect of the editorial letter by Mattioli et al. [2].

Ferri et al. suggested that tracheostomy should be performed in stable or clinically improved patients affected by Coronavirus Disease 2019 (COVID-19), not before the 20th day after orotracheal intubation. The authors support the "late tracheostomy protocol" thesis on 8 patients undergoing open tracheostomy declaring a negative prognostic trend for the subgroup of patients with shorter duration of intubation. We deem it inappropriate to draw conclusion with such a small cohort of patients and without any data and explanations regarding the reasons why an earlier tracheostomy would lead to worse survival.

Tracheostomy is one of the commonest surgical procedure in the setting of acute respiratory distress syndrome

This author's reply refers to the letter to the editor at doi: https:// doi.org/10.1007/s00405-020-06068-7.

This reply refers to the comment available online at https://doi. org/10.1007/s00405-020-06068-7.

Matteo Fermi

matteo.fermi.med@gmail.com

1 Otorhinolaryngology-Head and Neck Surgery Department, University Hospital of Modena, Via del Pozzo 71, 41100 Modena, Italy

2 Department of Anesthesiology, University Hospital of Modena, Via del Pozzo 71, 41100 Modena, Italy

3 Otorhinolaryngology-Head and Neck Surgery Department, University Hospital of Verona, Piazzale Aristide Stefani, 41100 Verona, Italy
(ARDS) with need of prolonged invasive mechanical ventilation (IMV). Traditionally, tracheostomies are performed to improve patient comfort, to facilitate airway and pulmonary toilet, to ease weaning and to facilitate the management of these patients. Moreover, it decreases the risk of complications such as subglottic and tracheal stenosis, which will likely become a striking reality amongst COVID-19 survivors (Mattioli et al.- under review). To date, one of the most convincing paper regarding this topic is the systematic review published by Adly et al. in 2018 [3]. The authors took into account 43 studies with a total number of 222,641 patients, showing that early tracheostomy (performed within 7 days from orotracheal intubation) reduces the clinical complications (i.e. aspiration pneumonia, bacteremia, septic shock, multi-organ failure), the mortality rate as well as the length of stay in intensive care unit (ICU).

Although delaying tracheostomy for patients with COVID-19 might reduce risks for staff, extended duration of translaryngeal intubation, sedation, IMV, and ICU stay associated with such delays can lead to complications [4].

Moreover, an increased need for ICU admission due to COVID-19-related ARDS was a reality at the time of the publication of Mattioli et al. editor letter [2]. The magnitude of this demand caused an imbalance between the real clinical needs of the population and the effective availability of intensive resources. Thus, the tracheostomy represented an important measure for early ICU discharge to intermediate care ward for patients with ongoing IMV. Recovering patients who continue to require ventilatory support via a tracheostomy can be managed with minimal sedation, which might simplify care and facilitate transfer to lower-acuity facilities, thus creating capacity for more acute patients. In accordance with recent expert's guidelines [4], we deem appropriate that standard decision making should be adapted for the COVID-19 pandemic, taking into account a range of considerations, including potential risks and benefits for the individual patient; risks posed to health-care workers, other 
Table 1 Factors related to the tracheostomy decision making in COVID-19 epidemic

\begin{tabular}{lc}
\hline Factors related to the tracheostomy in COVID-19 pandemic & \\
\hline Favors early tracheostomy & Favors delayed tracheostomy \\
\hline Maintenance of ICU capacity & $\begin{array}{c}\text { Potential risks to health-care } \\
\text { workers, patients, and family } \\
\text { Multiorgan failure and prognosis }\end{array}$ \\
Pulmonary hygiene & Requirement for prone ventilation \\
Laryngeal injury, trauma, or dysfunction & \\
Cumulative effects of sedation & \\
Expedited participation in rehabilitation & \\
Ventilator-associated pneumonia & \\
Ventilator-associated respiratory muscle atrophy & \\
Ability to communicate &
\end{tabular}

ICU Intensive Care Unit patients, and family; and available health-care resources (Table 1). The timing of tracheostomy should consider patient's clinical improvement perspective, which can be weighted through the Sequential Organ Failure Assessment (SOFA) score, as already stated in our previous Letter to the Editor and also demonstrated by other authors [5]. Although it is not possible to define an optimal timing, it is our opinion that tracheostomy should be indicated on case-by-case basis taking into account including potential risks and benefits for the individual patient; risks posed to health-care workers and other patients; and available health-care resources. In addition, we still deem appropriate to limit, when possible, late tracheostomies to avoid potential further complications.

Funding No funding is reported for the present study.

\section{Compliance with ethical standards}

Conflict of interest The authors declare no conflict of interest.

Ethical approval All the procedures performed in the study were in accordance with the ethical standards of the institutions at which the study was conducted.

\section{References}

1. Ferri E, Boscolo Nata F, Pedruzzi B et al (2020) Indications and timing for tracheostomy in patients with SARS CoV2-related. Eur Arch Otorhinolaryngol. https://doi.org/10.1007/s00405-02006068-7 (Published online May 26, 2020)

2. Mattioli F, Fermi M, Ghirelli M et al (2020) Tracheostomy in the COVID-19 pandemic. Eur Arch Otorhinolaryngol. https://doi. org/10.1007/s00405-020-05982-0

3. Adly A, Youssef TA, El-Begermy MM, Younis HM (2018) Timing of tracheostomy in patients with prolonged endotracheal intubation: a systematic review. Eur Arch Otorhinolaryngol 275(3):679-690. https://doi.org/10.1007/s00405-017-4838-7

4. McGrath BA, Brenner MJ, Warrillow SJ et al (2020) Tracheostomy in the COVID-19 era: global and multidisciplinary guidance. Lancet Respir Med. https://doi.org/10.1016/S2213 -2600(20)30230-7 (Published online May 2020)

5. Riestra-Ayora J, Yanes-Diaz J, Penuelas O, Molina-Quiros C, Sanz-Fernández R, Martin-Sanz E (2020) Safety and prognosis in percutaneous vs surgical tracheostomy in 27 patients With COVID-19. Otolaryngol Head Neck Surg. https://doi. org/10.1177/0194599820931801 (Published online May 26, 2020:194599820931801)

Publisher's Note Springer Nature remains neutral with regard to jurisdictional claims in published maps and institutional affiliations. 